\title{
IS THE CONSENSUS VALUE OF ANU SUCROSE (IAEA C-6) TOO HIGH?
}

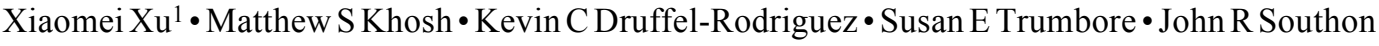 \\ Keck AMS Facility, Earth System Science Department, University of California, Irvine, California 92697, USA.
}

\begin{abstract}
Primary and secondary standards are essential in radiocarbon analyses for the purpose of reporting and comparing data among laboratories, as well as for internal laboratory data quality control. ANU sucrose is one of the IAEA-certified ${ }^{14} \mathrm{C}$ standards (C-6) with a consensus value of $1.5061 \pm 0.0011$ fraction modern (Fm). All of our measurements of ANU sucrose $(n=351)$ as a secondary standard over the last $7 \mathrm{yr}$ result in an average value of $1.5016 \pm 0.0005 \mathrm{Fm}(2-\sigma$ standard error). After applying the same outlier tests used for IAEA reference standards, a weighted average value of $1.5016 \pm 0.0002$ Fm $(n=294)$ was calculated. This value is significantly lower than the IAEA C-6 consensus value ( $t$ test with unequal variance; $p=0.023$ ). In contrast, our measurements of other secondary standards over the same time period are in excellent agreement with their respective consensus values. Since ANU is the only secondary standard measured in our lab that does not agree with the consensus values, and we have measured a larger number analyses compared to what went into the definition of the consensus value, we suggest that the consensus value of ANU sucrose might be too high by $\sim 0.0045 \pm 0.0011$ Fm. Given that some labs routinely use ANU sucrose as a primary standard, our results suggest that revisiting the consensus value of ANU sucrose may be necessary.
\end{abstract}

\section{INTRODUCTION}

ANU sucrose, produced from sugar cane grown between September 1965 and June 1971, is one of the IAEA-certified radiocarbon standards (C-6). The name ANU comes from the Australian National University (ANU); ANU sucrose was first supplied as a standard material by H A Polach (Polach 1979). There have been several rounds of international cross-calibration exercises involving ANU. The first reported value obtained from 15 radiocarbon laboratories and calibrated against NBS oxalic acid standard OXI was $1.5007 \pm 0.0052$ fraction modern (Fm) in 1979 (Polach 1979). The value was later modified to $1.5081 \pm 0.0020 \mathrm{Fm}$ in 1980 after more detailed examination (Currie and Polach 1980). The current consensus value is $1.5061 \pm 0.0011$ Fm (standard error; $n=22$ ) from the intercomparison exercise conducted in 1990 (Rozanski et al. 1992).

ANU is commonly used as a secondary ${ }^{14} \mathrm{C}$ standard by many laboratories, but is also preferred as a primary standard by some laboratories using liquid scintillation counting (LSC) systems because of its suitability for benzene preparation and relatively high activity compared to OXI. Advantages of using the ANU standard include the following: 1) A large quantity (2000 kg) has been carefully stored in drums; 2) It is analytically pure and relatively homogeneous; 3) It is chemically inert and relatively stable when dry and thus its exchange with atmospheric $\mathrm{CO}_{2}$ is minimal (Srdoc et al. 1979). In this paper, we report a discrepancy between the value obtained for ANU sucrose in our laboratory over the past $7 \mathrm{yr}$ (a total of 351 analyses) and the reported consensus value.

\section{METHODS}

\section{${ }^{14} \mathrm{C}$ Analysis}

Usually, $\sim 10 \mathrm{mg}$ of $\mathrm{ANU}$ is combusted with $\mathrm{CuO}$ in a sealed quartz tube at $900{ }^{\circ} \mathrm{C}$ for $2 \mathrm{hr}$, and $\mathrm{CO}_{2}$ is extracted cryogenically and split into four 1-mg $\mathrm{C}$ aliquots on a vacuum line. $\mathrm{CO}_{2}$ is then graphitized by the sealed-tube zinc reduction method (Xu et al. 2007; Khosh et al. 2010). Previous studies have reported no noticeable difference in ${ }^{14} \mathrm{C}$ results from $\mathrm{CO}_{2}$ graphitized directly from combusted 1-mg $\mathrm{C}$ samples and from splitting larger samples of $\mathrm{CO}_{2}$ into smaller aliquots (Southon and Santos

${ }^{1}$ Corresponding author. Email: xxu@uci.edu.

(C) 2010 by the Arizona Board of Regents on behalf of the University of Arizona Proceedings of the 20th International Radiocarbon Conference, edited by A J T Jull RADIOCARBON, Vol 52, Nr 2-3, 2010, p 866-874 
2004; Xu et al. 2007). ${ }^{14} \mathrm{C}$ analysis was conducted at the Keck Carbon Cycle AMS facility at the University of California, Irvine (KCCAMS), where the system is a compact accelerator mass spectrometer (AMS) from National Electrostatics Corporation (NEC 0.5MV 1.5SDH-2 AMS system) with a modified NEC MC-SNIC ion source (Southon and Santos 2004, 2007). The in situ simultaneous AMS $\delta^{13} \mathrm{C}$ measurement at KCCAMS allowed for the correction of fractionation occurring both during the graphitization process and inside the AMS system, and thus significantly improved the precision and accuracy of our measurements.

We use OXI as our primary standard, with 6-7 OXI standards in each 40-sample wheel. We routinely have 3-4 secondary standards in each wheel, usually 1 each of the ANU and OXII standards, and 1 of FIRI-J or TIRI-B depending on the ${ }^{14} \mathrm{C}$ content of the samples we measure. These secondary standards are used to determine the overall accuracy of the samples prepared and measured in our laboratory. With this approach, the relative percent error of our day-to-day analysis, including combustion, graphitization, and AMS measurement, is between 2.5 to $3.1 \%$ for all of our secondary standards processed in the last $7 \mathrm{yr}$ (see Table 2).

\section{IAEA C-6 Data Set and ANU Values from Other Labs}

We obtained the IAEA C-6 data set for the 1990 intercomparison exercise from the IAEA consultants group meeting report by Rozanski (1991). In this report, only the results of those laboratories that agreed to disclose their identity are listed. We found 32 values out of 39 labs that returned their ${ }^{14} \mathrm{C}$ measurements for C-6. This data set was used to compare with the UCI C-6 data set given below. In the meantime, other investigators shared their results with us when requested for comparison of ANU values from other ${ }^{14} \mathrm{C}$ or graphite preparation laboratories.

\section{Outlier Tests}

During the 1990 intercomparison exercise, the data analysis and the consensus value calculation were presented by Rozanski et al. (1992). In this exercise, 2 outlier tests were conducted on the original data set, before the consensus value calculation, in order to obtain an unbiased estimate of the consensus value:

Outlier test 1: Values are omitted if they are $<H_{L}-3.0 \times\left(H_{U}-H_{L}\right)$ or $>$ than $H_{L}+3.0 \times\left(H_{U}-H_{L}\right)$, where $H_{L}$ and $H_{U}$ are the lower and upper quartiles, respectively.

Outlier test 2: $|(x-m) / s|<2$, where $x$ is the ${ }^{14} \mathrm{C}$ result $(\mathrm{Fm}), s$ the quoted error, and $m$ the median found after test 1 . This test removes results with quoted errors that are either unrealistically small or large.

The outlier removal process used is very important because averages are sensitive to extreme data values. These same outlier tests were also applied to the TIRI and FIRI intercomparison exercises (Scott 2003c). In order to compare our UCI ANU data set with the IAEA C-6 data set, we applied the same outlier tests to our data set.

\section{Consensus Value Evaluation}

The consensus value is calculated as a weighted average $\left(X_{w}\right)$ of the filtered data set (see Equation 1) and the uncertainty is calculated as the estimated standard error (Equation 2), following the method described by Rozanski et al. (1992) and later by Scott (2003c): 


$$
\begin{gathered}
X_{w}=\frac{\left(\sum \frac{X_{i}}{W_{i}^{2}}\right)}{\left(\sum \frac{1.0}{W_{i}^{2}}\right)} \\
\text { ese }=\sqrt{\frac{\frac{1}{n}\left(\sum \frac{X_{i}-X_{w}^{2}}{W_{i}^{2}}\right)}{\left(\sum \frac{1.0}{W_{i}^{2}}\right)}}
\end{gathered}
$$

where $X_{i}$ and $W_{i}$ are the individual result and quoted error, respectively, and $n$ is the total number of data points used in the weighted average calculation after the 2 outlier tests.

\section{Statistics}

We applied a $t$ test with unequal variances to test for significance of differences between the means and weighted means for our UCI ANU data set and the IAEA C-6 data sets before and after outlier removal tests.

\section{RESULTS}

Individual measurements of ANU sucrose and OXII secondary standards are plotted in Figure 1. The mean of all measurements was $1.5016 \pm 0.0005 \mathrm{Fm}$ for ANU $(2-\sigma$ standard error; $n=351)$ and $1.3398 \pm 0.0003 \mathrm{Fm}$ for OXII (2- $\sigma$ standard error; $n=550)$. We give results for FIRI-J and TIRI-B standards in Table 1. The ANU value is below the consensus value (Figure 1a), while that for OXII is in accord with its consensus value (Figure 1b).

Table 1 Comparisons of all lab measured secondary standards to their consensus values. All show excellent agreements except for ANU sucrose.

\begin{tabular}{llllc}
\hline Standard & ANU $(\mathrm{Fm})$ & OXII $(\mathrm{Fm})$ & FIRI-J $(\mathrm{Fm})$ & TIRI-B $(\mathrm{yr})$ \\
\hline Consensus value & $1.5061 \pm 0.0011$ & $1.3407 \pm 0.0019$ & $1.1069 \pm 0.0004$ & $4508 \pm 3$ \\
Lab average & 1.5016 & 1.3398 & 1.1069 & 4521 \\
Standard deviation & 0.0046 & 0.0041 & 0.0027 & 25 \\
\% error & 0.30 & 0.31 & 0.25 & 0.54 \\
$n$ & 351 & 602 & 165 & 198 \\
\hline
\end{tabular}

Figure 2 shows the boxplots for comparison of our UCI ANU and OXII data sets before and after the IAEA outlier tests. The outlier tests did not change the mean value for either ANU or OXII standards and failed to make the UCI ANU data set agree with the ANU consensus value. The calculated weighted average of UCI ANU data set is $1.5016 \pm 0.0002 \mathrm{Fm}$, from 294 data points that passed the 2 outlier tests. The $t$ test results indicate that the means of our UCI ANU data set and the IAEA C-6 data set are not significantly different after only outlier test $1(P=0.60$ for $(T \geq t) 2$ tails), but the difference become significant after outlier test $2(P=0.023)$. 
(a)

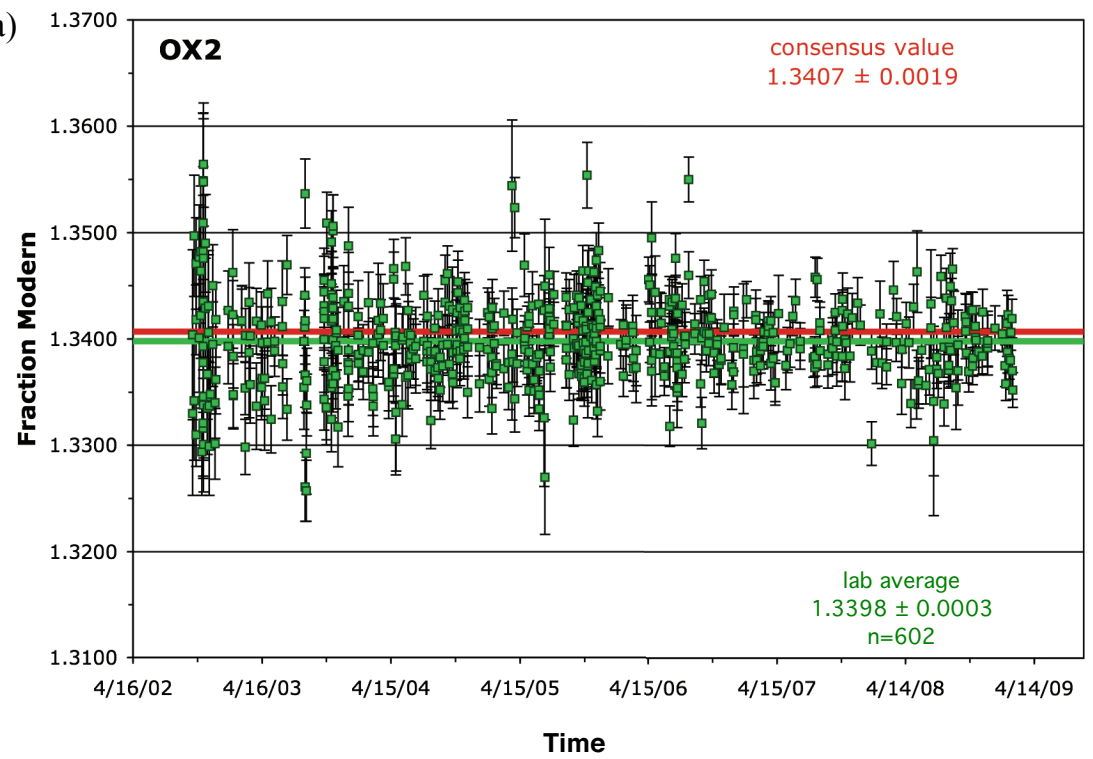

(b)

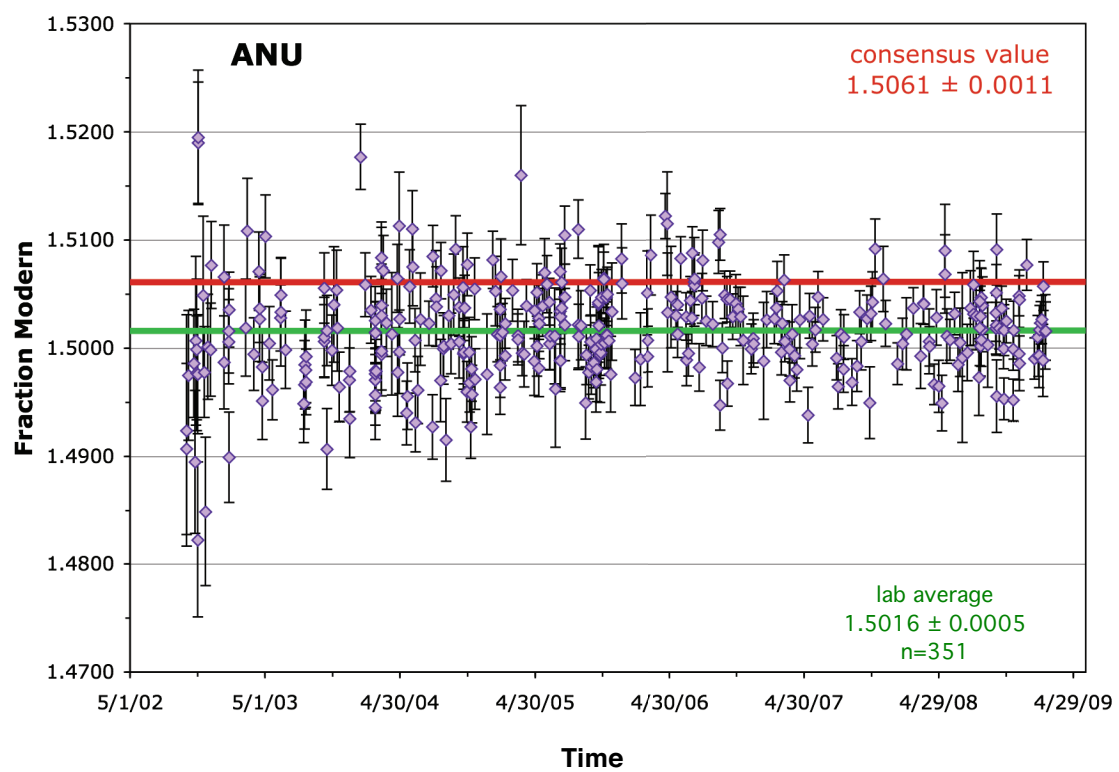

Figure 1 (a) All ANU measurements in the last $7 \mathrm{yr}$. The red line indicates the average of all data points, while the green line represents the consensus value of C-6. Our lab average appears to be lower than the IAEA consensus value. (b) All OXII measurements in the last $7 \mathrm{yr}$. The lab average of all OXII data points agrees very well with OXII consensus value.

Figure 3 shows relative frequency plots of the IAEA C- 6 and UCI ANU data sets, both raw and filtered data using the IAEA outlier removal tests. Obviously, the IAEA data set is smaller and more scattered because it came from a few dozens of different laboratories. Out of the filtered IAEA C-6 data set, 10 results were from LSC labs, 9 were from gas proportional counting (GPC) labs, and only 3 were from the AMS labs. Figure 3 also shows our ANU data is normally distributed. 
(a) ANU

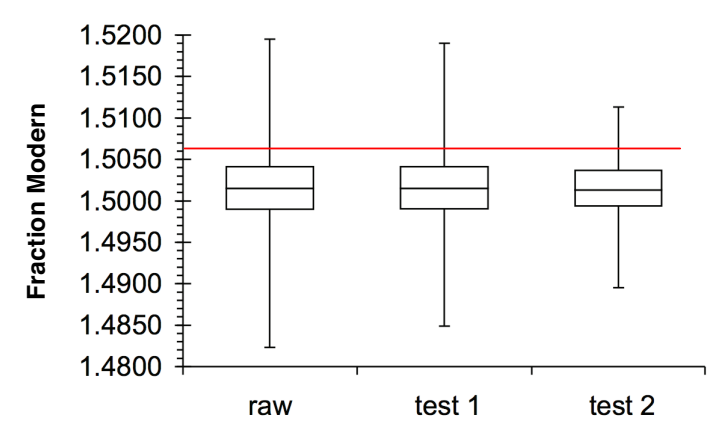

(b) OXII

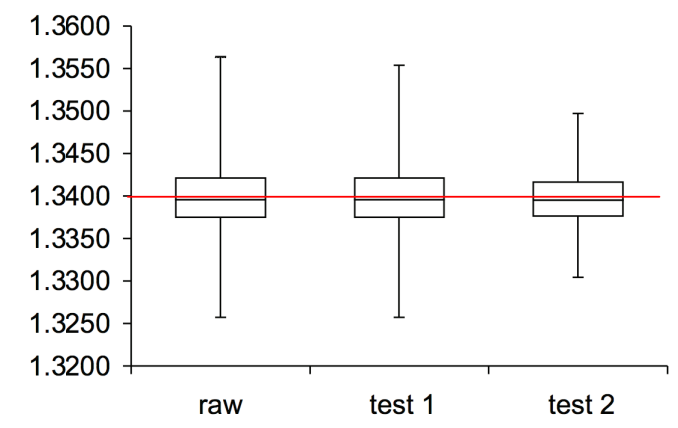

Figure 2 Boxplots for comparison of UCI ANU (a) and OXII (b) data sets before and after the IAEA 2 outlier tests. The outlier removal processes failed to make the UCI ANU data set agree with the consensus value indicated by the red line, while the UCI OXII data set agrees well with its consensus value whether the data are filtered or not.

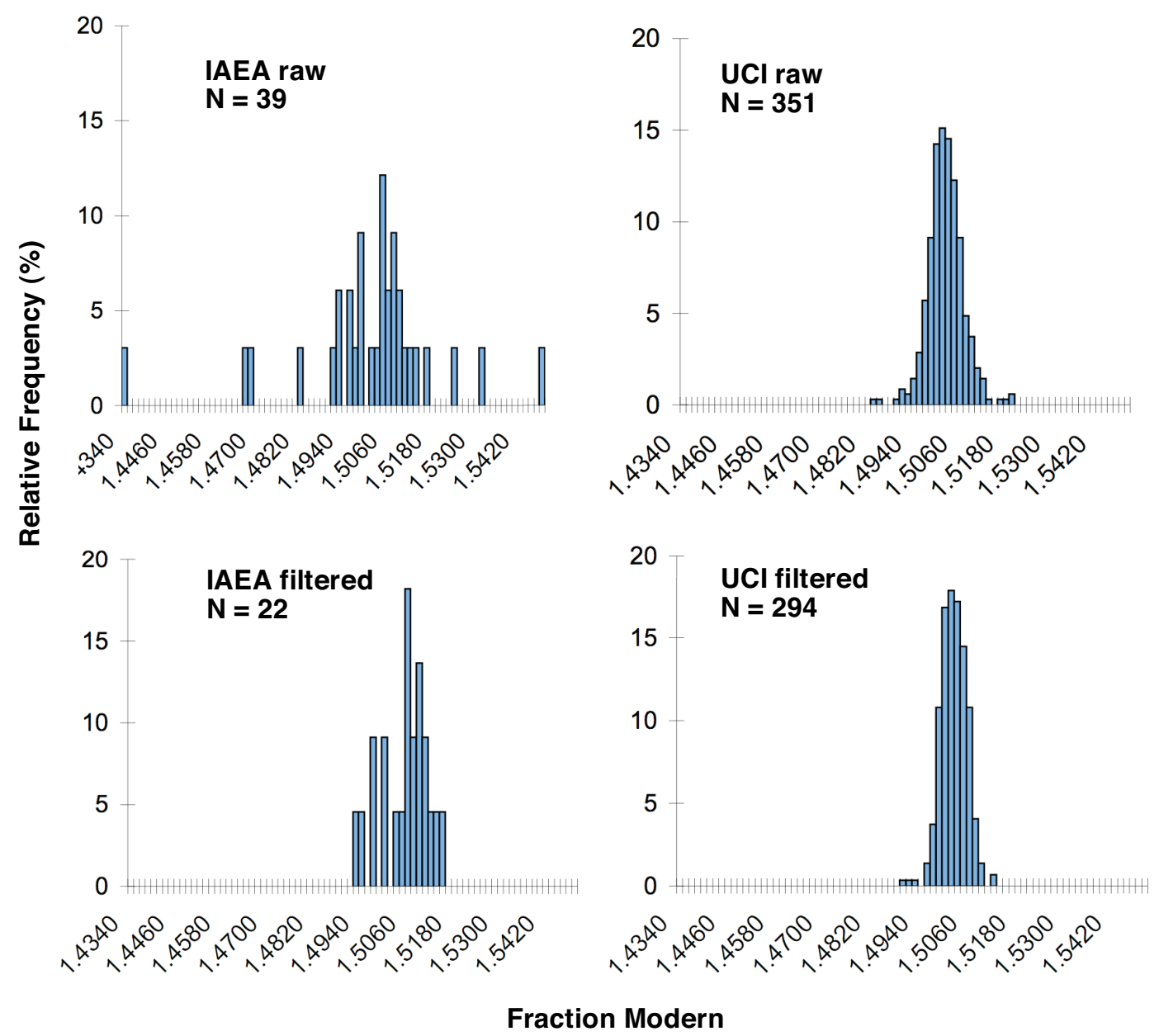

Figure 3 Relative frequency plots of IAEA and UCI data sets for ANU, for all data, and data filtered using IAEA criteria. The IAEA data set is much smaller and more scattered. 
Figure 4 shows the plots of $\mathrm{Fm} \pm 2$ quoted errors for all data points from our UCI ANU data set and the IAEA C-6 data set used to define the suggested consensus values. Figure 4a clearly shows that the calculated UCI ANU weighted average (red line) is lower than the IAEA C-6 consensus value (green line).
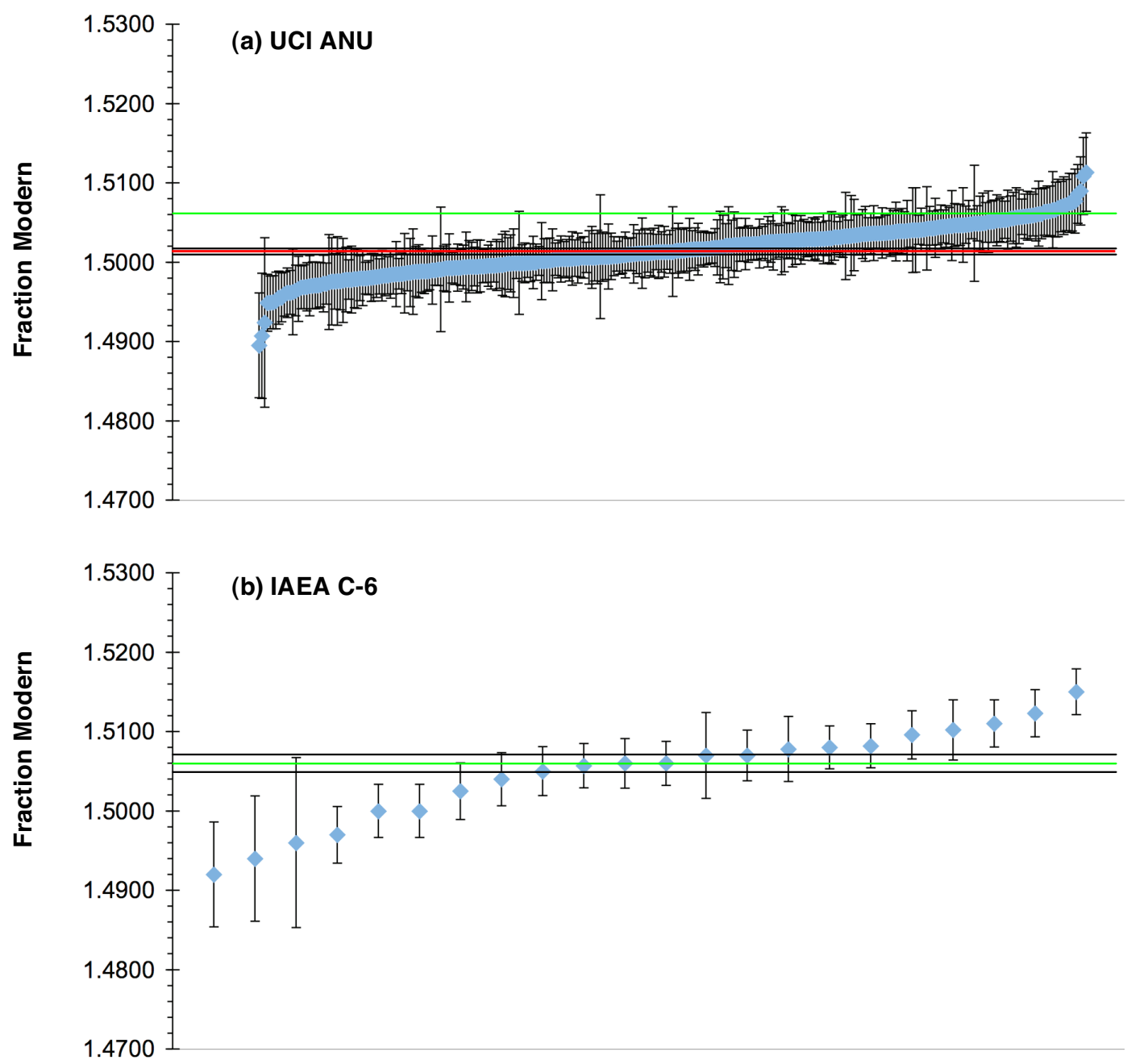

Figure 4 Plot of fraction modern $(\mathrm{Fm}) \pm 2$ quoted errors for all UCI ANU values (a) and all IAEA C-6 values (b) used to evaluate the consensus values. The green line is the IAEA C- 6 consensus value and the red line is the suggested "consensus" value of $1.5016 \pm 0.0002$ by the UCI ANU data set using the same weighted estimation of IAEA (Rozanski et al. 1992; Scott 2003c).

\section{DISCUSSION}

\section{Comparison with Other Laboratories}

Our data clearly demonstrate that the ANU sucrose measured in our laboratory has a lower ${ }^{14} \mathrm{C}$ value than the consensus value. The fact that all other secondary standards in our laboratory are in agreement with their respective consensus values leads us to 2 possibilities: 1) that the ANU sucrose stan- 
dard is not homogeneous, and our aliquot is not representative; or 2) that the consensus value presently reported is too high. To test the first possibility, we collected ANU measurements from 9 other ${ }^{14} \mathrm{C}$ labs and graphite preparation labs to see if their results are consistent with ours (Table 2). The collected average values (not filtered) range from 1.5012 to $1.05042 \mathrm{Fm}$, with an unweighted mean of $1.5023 \pm 0.0013 \mathrm{Fm}$. This agrees very well with our slightly lower value of $1.5016 \mathrm{Fm}$. Most of the labs listed in Table 2 used AMS for ${ }^{14} \mathrm{C}$ measurement. A few labs listed here have their graphite measured in the UCI AMS facility; therefore, their results may not be completely independent from ours. However, some of the labs utilized different graphitization methods, different aliquots of the ANU sucrose, or used OXII instead of OXI as the primary standard. Four labs acquired their ANU sucrose from the IAEA as C-6, while others may have obtained the material from the Australian National University directly or indirectly at different times. There seems to be no difference between IAEA C-6 and ANU acquired from the Australian National University, indicating that ANU sucrose is relatively homogeneous.

Table 2 Comparisons of ANU measurements from other graphite preparation labs and other ${ }^{14} \mathrm{C}$ labs. All collected average values of ANU from other labs agree with our slightly lower lab average value.

\begin{tabular}{|c|c|c|c|c|c|c|c|c|}
\hline Laboratory & $\begin{array}{l}\text { Primary } \\
\text { std }\end{array}$ & Graphitization & $\begin{array}{l}{ }^{14} \mathrm{C} \\
\text { meas }\end{array}$ & $n$ & $\begin{array}{l}\text { Average } \\
\text { fM }\end{array}$ & St dev & From & Source \\
\hline UCI/Trumbore-Xu & OXI & $\begin{array}{l}\text { Sealed-tube } \\
\mathrm{Zn} \text { reduction }\end{array}$ & $\begin{array}{l}\text { UCI } \\
\text { AMS }\end{array}$ & 351 & 1.5016 & 0.0040 & ANU & This study \\
\hline UCI/Santos-Southon & OXI & $\mathrm{H}_{2}$ reduction & $\begin{array}{l}\text { UCI } \\
\text { AMS }\end{array}$ & 119 & 1.5012 & 0.0052 & ANU & pers. comm. \\
\hline UCI/Druffel-Griffin & OXI & $\mathrm{H}_{2}$ reduction & $\begin{array}{l}\text { UCI } \\
\text { AMS }\end{array}$ & 35 & 1.5042 & 0.0061 & ANU & pers.comm. \\
\hline Univ of Florida/Schuur & OXII & $\mathrm{H}_{2}$ reduction & $\begin{array}{l}\text { UCI } \\
\text { AMS }\end{array}$ & 57 & 1.5031 & 0.0059 & C-6 & pers. comm. \\
\hline Univ of Bayreuth/Borken & OXII & $\begin{array}{l}\text { Sealed-tube } \\
\text { Zn reduction }\end{array}$ & $\begin{array}{l}\text { UCI } \\
\text { AMS }\end{array}$ & 46 & 1.5014 & 0.0044 & C-6 & pers. comm. \\
\hline Waikato/Hogg & OXII & & LSC & 2 & 1.50305 & 0.00007 & ANU & pers. comm. \\
\hline Groningen/van der Plicht & OXI & $\mathrm{H}_{2}$ reduction & AMS & 18 & 1.5030 & 0.0030 & ANU & pers. comm. \\
\hline Groningen/van der Plicht & OXI & & GPC & many & 1.5036 & 0.0040 & ANU & pers. comm. \\
\hline Univ of Utah/Ehleringer & OXII & $\begin{array}{l}\text { Sealed-tube } \\
\text { Zn reduction }\end{array}$ & $\begin{array}{l}\text { UCI } \\
\text { AMS }\end{array}$ & 20 & 1.5013 & 0.0028 & C-6 & pers. comm. \\
\hline NOSAMS/McNichol & OXI & $\mathrm{H}_{2}$ reduction & AMS & 50 & 1.4999 & 0.0097 & ANU & pers. comm. \\
\hline SUERC/Xu & OXI & $\mathrm{Zn}$ reduction & AMS & 5 & 1.5031 & 0.0039 & C-6 & Xu et al. 2004 \\
\hline
\end{tabular}

A weighted average from all values in Table 2 is $1.5017 \mathrm{Fm}$. If only values from other labs are considered, the weighted average is $1.5018 \mathrm{Fm}$. Both of these values are consistent with our weighted average of $1.5016 \mathrm{Fm}$, calculated using the IAEA approach after outlier removal, and the average without outlier removal (1.5016 Fm).

\section{Possible Reasons for the Discrepancy Observed}

1) The IAEA data set is relatively small and scattered. Rozanski et al. (1992) stated C-6 shows much lower precision than the other samples (a combination of larger errors and fewer labs). Although 39 labs returned their ANU measurement results in the 1990 intercomparison exercise, only 22 labs' results passed the 2 outlier tests ( $44 \%$ of the labs failed the tests). Furthermore, the 22 points remaining after outlier were removed are still quite scattered with a standard deviation (st dev) of $\pm 0.0064 \mathrm{Fm}$. This is probably due to the fact that the majority of the labs among these 22 labs were LSC or GPC types (19 out 22), and data from these labs were often found to be more scattered than AMS labs (Rozanski et al. 1992; Scott 2003a,c,d). During recent years, the high sample throughput made possible by AMS allows for more precise com- 
parison of ANU with OXI than has been possible in the past. The use of simultaneously measured $\mathrm{AMS}{ }^{13} \mathrm{C} /{ }^{12} \mathrm{C}$ for fractionation correction has also significantly improved the precision and accuracy of AMS measurements because it can account for fractionation processes that occur during graphitization and inside the AMS.

2) The underlying assumption that all the data in the IAEA interlaboratory comparison (in 1990 exercise) represented a statistical population without remaining biases was not fully achieved.

3) ANU is not homogenous. This probably is unlikely, both from the results in Table 2 and the $\delta^{13} \mathrm{C}$ measurement. A portion of the ANU original material was checked for isotopic homogeneity and was later renamed to IAEA-CH- 6 as a ${ }^{13} \mathrm{C}$ international standard. Its recommended $\delta^{13} \mathrm{C}$ value was determined to be $-10.449 \pm 0.033 \%$ (versus VPDB) in an international calibration exercise (Coplen et al. 2006).

4) The UCI data set is systematically off due to unknown bias. This is also unlikely because other secondary standards run in the same time interval do not show any systematic offsets from their respective consensus values (Table 1).

\section{SUMMARY}

Our large data set using $7 \mathrm{yr}$ of AMS measurements suggests the value for ANU is slightly different from the consensus value, on the order of $0.0045 \pm 0.0011 \mathrm{Fm}$ too low. The difference is statistically significant. Consistent agreement of our other secondary standards (especially OXII) with their consensus values suggests the deviation in our ANU value from the IAEA C- 6 consensus value is real.

Measurements of ANU from many other graphite preparation labs and ${ }^{14} \mathrm{C}$ labs agree with our result and indicate a Fm value that is lower than the IAEA C- 6 consensus value. Given that some labs routinely use ANU sucrose as a primary standard, our results suggest that revisiting the consensus value of ANU sucrose may be necessary. With many more AMS laboratories currently in operation than $20 \mathrm{yr}$ ago and many of them able to produce high-precision data (2-3\%), we should be able to get a more accurate estimate of the ${ }^{14} \mathrm{C}$ value for ANU sucrose.

\section{ACKNOWLEDGMENTS}

We thank the W M Keck foundation, the NSF Carbon Cycle Program, and UC Irvine for financial support. We are grateful to Drs Alan Hogg, Hans van der Plicht, Ann McNicol, Guaciara dos Santos, Ted Schuur, Werner Borken, Jan Muhr, James Ehleringer, Ellen Druffel, and Sheila Griffin who provided us their ANU values for comparison. Special thanks go to Dr Manfred Gröning, the Head of Isotope Hydrology Laboratory of IAEA, for his insightful comments. We are also grateful to 2 referees for their helpful reviews.

\section{REFERENCES}

Coplen TB, Brand WA, Gehre M, Gröning M, Meijer HAJ, Toman B, Verkouteren RM. 2006. New guidelines for $\delta^{13} \mathrm{C}$ measurements. Analytical Chemistry 78(7):2439-41.

Currie LA, Polach HA. 1980. Exploratory analysis of the international radiocarbon cross-calibration data: consensus values and interlaboratory error. Radiocarbon 22(3):933-5.

Khosh MS, Xu X, Trumbore SE. 2010. Small-mass graphite preparation by sealed tube zinc reduction method for AMS ${ }^{14} \mathrm{C}$ measurements. Nuclear Instruments and Methods in Physics Research B 268(7-8):927-30.

Polach HA. 1979. Correlation of ${ }^{14} \mathrm{C}$ activity of NBS ox- alic acid with Arizona 1850 wood and ANU sucrose standards. In: Berger R, Suess HE, editors. Radiocarbon Dating. Proceedings of the 9th International ${ }^{14} \mathrm{C}$ Conference. Berkeley: University of California Press. p 115-24.

Rozanski K. 1991. International Atomic Energy Agency Consultants' Group Meeting on C-14 Reference Materials for Radiocarbon Laboratories, 18-20 February 1991. Section of Isotope Hydrology, IAEA, Vienna.

Rozanski K, Stichler W, Gonfiantini R, Scott EM, Beukens RP, Kromer B, van der Plicht J. 1992. The IAEA ${ }^{14} \mathrm{C}$ Intercomparison Exercise 1990. Radiocarbon 34(3):506-19. 
SScott EM. 2003a. Section 4: Investigation of potential sources of variation. Radiocarbon 45(2):175-212.

Scott EM. 2003b. Section 6: Kauri wood, sample A and B. Radiocarbon 45(2):227-48.

Scott EM. 2003c. Section 7: Characterization of the reference materials by consensus values. Radiocarbon 45(2):249-67.

Scott EM. 2003d. Section 10: Summary and conclusions. Radiocarbon 45(2):285-90.

Southon JR, Santos GM. 2004. Ion source development at KCCAMS, University of California, Irvine. Radiocarbon 46(1):33-9.

Southon JR, Santos GM. 2007. Life with MC-SNICS. Part II: further ion source development at the Keck Carbon Cycle AMS facility. Nuclear Instruments and
Methods in Physics Research B 259(1):88-93.

Srdoc D, Obelic B, Horvatincic N, Sliepcevic A. 1979. Measurement of the ${ }^{14} \mathrm{C}$ activity of the ANU sucrose secondary standard by means of the proportional counter technique. Radiocarbon 21(3):321-8.

Xu S, Anderson R, Bryant C, Cook GT, Dougans A, Freeman S, Naysmith P, Schnabel C, Scott EM. 2004. Capabilities of the new SUERC 5MV AMS facility for ${ }^{14} \mathrm{C}$ dating. Radiocarbon 46(1):59-64.

Xu X, Trumbore SE, Zheng S, Southon JR, McDuffee KE, Luttgen M, Liu JC. 2007. Modifying a sealed tube zinc reduction method for preparation of AMS graphite targets: reducing background and attaining high precision. Nuclear Instruments and Methods in Physics Research B 259(1):320-9. 